\title{
PERMANENT GENETIC RESOURCES NOTE Permanent Genetic Resources added to Molecular Ecology Resources Database 1 December 2010-31 January 2011
}

Molecular Ecology Resources Primer Development Consortium, ${ }^{1}$ KiYOKaZU AgAtA, ${ }^{2}$ SAMER ALASAAD, ${ }^{3,4}$ VERA MARIA FONSECA ALMEIDA-VAL, ${ }^{5} \mathrm{~J}$. A. ÁLVAREZ-DIOS, ${ }^{6}$ F. BARBISAN, ${ }^{7}$ JON S. BEADELL,${ }^{8} \mathrm{~J}$. F. BELTRÁN, ${ }^{9} \mathrm{M}$. BENÍTEZ, ${ }^{10} \mathrm{G}$. BINO,${ }^{7}$ COLIN BLEAY, ${ }^{11}$ P. BLOOR, ${ }^{12}$ JÖRG BOHLMANN, ${ }^{13}$ WARREN BOOTH, ${ }^{14}$ E. BOSCARI, ${ }^{7}$ ADALGISA CACCONE, ${ }^{8}$ TATIANA CAMPOS, ${ }^{15}$ B. M. CARVALHO,${ }^{16,17}$ GISELE TORRES CLIMACO,${ }^{5}$ JEAN CLOBERT, ${ }^{11}$ L. CONGIU, ${ }^{7}$ CHRISTINA COWGER,${ }^{18}$ G. DIAS, ${ }^{16,17}$ I. DOADRIO, ${ }^{19}$ IZENI PIRES FARIAS, ${ }^{20}$ N. FERRAND ${ }^{16,17}$ PATRÍCIA D. FREITAS, ${ }^{21}$ G. FUSCO ${ }^{7}$ PEDRO M. GALETTI ${ }^{21}$ CRISTIAN GALLARDO-ESCÁRATE, ${ }^{22}$ MICHAEL W. GAUNT, ${ }^{23}$ ZANELI GOMEZ OCAMPO, ${ }^{8}$ H. GONÇALVES, ${ }^{16}$ E. G. GONZALEZ, ${ }^{24}$ PILAR HAYE ${ }^{25}$ O. HONNAY, ${ }^{26}$ CHAZ HYSENI, $^{8}$ H. JACQUEMYN, ${ }^{26}$ MICHAEL J. JOWERS, ${ }^{3}$ AKIHIRO KAKEZAWA, ${ }^{2}$ ERI KAWAGUCHI, ${ }^{2}$ CHRISTOPHER I. KEELING, ${ }^{13}$ YE-SEUL KWAN, ${ }^{27}$ MICHELANGELO LA SPINA, ${ }^{28}$ WAN-OK LEE ${ }^{29}$ M. LEŚNIEWSKA, ${ }^{30}$ YANG LI, ${ }^{31,32}$ HAIXIA LIU, ${ }^{31}$ XIAOLIN LIU, ${ }^{31}$ S. LOPES, ${ }^{16}$ P. MARTÍNEZ, ${ }^{33}$ S. MEEUS, ${ }^{26}$ BRENT W. MURRAY, ${ }^{34}$ ALINE G. NUNES, ${ }^{21}$ LOYCE M. OKEDI, ${ }^{35}$ JOHNSON O. OUMA, ${ }^{36}$ B. G. PARDO,${ }^{33}$ RYAN PARKS,${ }^{18}$ MARIA NAZARÉ PAULA-SILVA, ${ }^{5}$ C. PEDRAZA-LARA, ${ }^{19}$ OMATHTHAGE P. PERERA, ${ }^{37}$ A. PINO-QUERIDO, ${ }^{33}$ MURIELLE RICHARD, ${ }^{38}$ BRUNO C. ROSSINI, ${ }^{21}$ N. GAYATHRI SAMARASEKERA, ${ }^{34}$ ANTONIO SÁNCHEZ, ${ }^{4}$ JUAN A. SANCHEZ, ${ }^{28}$ CARLOS HENRIQUE DOS ANJOS SANTOS, ${ }^{5}$ WATARU SHINOHARA, ${ }^{2}$ RAMÓN C. SORIGUER, ${ }^{3}$ ADNA CRISTINA BARBOSA SOUSA, ${ }^{15}$ CAROLINA FERNANDES DA SILVA SOUSA, ${ }^{5}$ VIRGINIE M. STEVENS, ${ }^{39} \mathrm{M}$. TEJEDO, ${ }^{40}$ MYRIAM VALENZUELA-BUSTAMANTE, ${ }^{22} \mathrm{M}$. S. VAN DE VLIET, ${ }^{41} \mathrm{~K}$. VANDEPITTE, ${ }^{26} \mathrm{M}$. VERA, ${ }^{33}$ PETER WANDELER, ${ }^{42}$ WEIMIN WANG, ${ }^{32}$ YONG-JIN WON, ${ }^{27}$ A. YAMASHIRO, ${ }^{43}$ T. YAMASHIRO $^{44}$ and CHANGCHENG ZHU ${ }^{45}$

${ }^{1}$ Molecular Ecology Resources Editorial Office, 6270 University Blvd, Vancouver, BC, V6T 1Z4, Canada, ${ }^{2}$ Graduate School of Science, Kyoto University, Kyoto 606-8502, Japan, ${ }^{3}$ Estación Biológica de Doñana, Consejo Superior de Investigaciones Científicas (CSIC), Avda. Américo Vespucio s/n, 41092 Seville, Spain, ${ }^{4}$ Departamento de Biología Experimental, Universidad de Jaén, Campus Las Lagunillas, s/n, E-23071 Jaén, Spain, ${ }^{5}$ Instituto Nacional de Pesquisas da Amazônia (INPA), Laboratório de Ecofisiologia e Evolução Molecular, Manaus, AM, CEP 69060-001, Brazil, ${ }^{6}$ Departamento de Matemática Aplicada, Facultad de Matemáticas, Universidad de Santiago de Compostela, 15782 Santiago de Compostela, Spain, ${ }^{7}$ Department of Biology, University of Padova, via Ugo Bassi 58/B, I-35121 Padova, Italy, ${ }^{8}$ Department of Ecology and Evolutionary Biology, Yale University, 21 Sachem Street, New Haven, CT 06511, USA, ${ }^{9}$ Departamento de Fisiología y Zoología, Universidad de Sevilla, Avda. Reina Mercedes, 6, 41012 Sevilla, Spain, ${ }^{10}$ Departamento de Biología Animal, Facultad de Ciencias, Universidad de Granada, Campus Fuente Nueva s/n, 18071 Granada, Spain, ${ }^{11}$ CNRS USR 2936, Station d'Ecologie Expérimentale du CNRS à Moulis, 09200 Moulis, France, ${ }^{12}$ Grupo de Biodiversidad y Recursos Genéticos, Instituto de Genética, Universidad Nacional de Colombia, Bogotá, Colombia, ${ }^{13}$ Michael Smith Laboratories, University of British Columbia, 321-2185 East Mall, Vancouver, BC, V6T 1Z4, Canada, ${ }^{14}$ Department of Entomology and W.M. Keck Centre for Behavioral Biology, North Carolina State University, Campus Box 7613, Raleigh, NC 27695-7613, USA, ${ }^{15} 210$ Universidade Estadual de Campinas (UNICAMP), Centro de Biologia Molecular e Engenharia Genética (CBMEG), Campinas, SP, CP 6109, CEP 13083-970, Brazil, ${ }^{16}$ CIBIO, Centro de Investigação em Biodiversidade e Recursos Genéticos, Campus Agrário de Vairão, 4485-661 Vairão, Portugal, ${ }^{17}$ Departamento de Zoologia e Antropologia, Faculdade de Ciências da Universidade do Porto, Rua do Campo Alegre, s/n, 4169-007 Porto, Portugal, ${ }^{18}$ USDA-ARS, Department of Plant Pathology, North Carolina State University, Raleigh, NC 27695, USA, ${ }^{19}$ Departamento de Biodiversidad y Biología Evolutiva, Museo Nacional de Ciencias Naturales, CSIC, José Gutiérrez Abascal 2, 28006 Madrid, Spain, ${ }^{20}$ Universidade Federal do Amazonas (UFAM), Laboratório de Evolução e Genética Animal Manaus, AM, CEP 69077-000, Brazil, ${ }^{21}$ Departamento de Genética e Evolução, Universidade Federal de São Carlos, Via Washington Luiz, Km 235, PO Box 676, CEP 13565-905, São Carlos, SP, Brazil, ${ }^{22}$ Laboratorio de Genética y Biotecnología Acuícola, Centro de Biotecnología, Universidad de Concepción, Concepción, Chile, ${ }^{23}$ Infectious and Tropical Diseases Department, London School of Hygiene and Tropical Medicine, Keppel Street, London WC1E 7HT, UK, ${ }^{24}$ Departamento de Bioquímica y Biología Molecular IV , Universidad Complutense de Madrid (UCM), Facultad de Veterinaria, 28040 Madrid, Spain, ${ }^{25}$ Laboratorio de Diversidad 
Molecular, Departamento de Biología Marina, Universidad de Católica del Norte, Coquimbo, Chile, ${ }^{26}$ Laboratory of Plant Ecology, University of Leuven, Kasteelpark Arenberg 31, 3001 Heverlee, Belgium, ${ }^{27}$ Division of EcoScience, Ewha Womans University, Science B Building (rm 554), 11-1 Daehyon-Dong, Sodaemun-Ku, Seoul, 120-750, Korea, ${ }^{28}$ Instituto Murciano de Investigación y Desarrollo Agrario y Alimentario (IMIDA), C/Mayor, 1, La Alberca, 30150 Murcia, Spain, ${ }^{29}$ Inland Fisheries Research Institute, National Fisheries Research \& Development Institute, Gapyeong, Gyeonggi-do, 444-815, Korea, ${ }^{30}$ Department of General Zoology, A. Mickiewicz University, Umultowska 89, 61-614, Poznań, Poland, ${ }^{31}$ College of Animal Science and Technology, Northwest A E F University, 712100 Yangling, Shanxi, China, ${ }^{32}$ College of Fisheries, Huazhong Agricultural University, 430070 Wuhan, Hubei, China, ${ }^{33}$ Departamento de Genética, Facultad de Veterinaria, Universidad de Santiago de Compostela, Campus de Lugo, 27002 Lugo, Spain, ${ }^{34}$ Natural Resources and Environmental Studies Institute, University of Northern British Columbia, 3333 University Way, Prince George, BC, V2N 4Z9, Canada, ${ }^{35}$ National Livestock Resources Research Institute, P.O. Box 96, Tororo, Uganda, ${ }^{36}$ Trypanosomiasis Research Centre, Kenya Agricultural Research Institute, P.O. Box 362-00902, Kikuyu, Kenya, ${ }^{37}$ Southern Insect Management Research Unit, USDA-ARS, Stoneville, MS 38776, USA, ${ }^{38}$ CNRS UMR 7625, Université Pierre et Marie Curie, Case 237, 7 Quai St Bernard, 75005 Paris, France, ${ }^{39}$ FRS-FNRS, University of Liège, 22 Quai VanBeneden, 4020 Liège, Belgium, ${ }^{40}$ Department of Evolutionary Ecology, Estación Biológica de Doñana, CSIC, Avda. Americo Vespucio, s/n, 41092 Sevilla, Spain, ${ }^{41}$ CCMAR, FCMA, Universidade do Algarve, Gambelas, 8005-139 Faro, Portugal, ${ }^{42}$ Institute of Evolutionary Biology and Environmental Studies (IEU), University of Zürich, Winterthurerstrasse 190, 8057 Zürich, Switzerland, ${ }^{43}$ Graduate School Advanced Technology and Science, The University of Tokushima, 2-1 Minami-josanjima, Tokushima 770-8506, Japan, ${ }^{44}$ Environmental Symbiosis Studies, Graduate School of Integrated Arts and Sciences, The University of Tokushima, 1-1 Minami-josanjima, Tokushima 770-8502, Japan, ${ }^{45}$ Department of Biology, Kim II-sung University, Taesong District, Pyongyang, Korea

\begin{abstract}
This article documents the addition of 238 microsatellite marker loci to the Molecular Ecology Resources Database. Loci were developed for the following species: Alytes dickhilleni, Arapaima gigas, Austropotamobius italicus, Blumeria graminis f. sp. tritici, Cobitis lutheri, Dendroctonus ponderosae, Glossina morsitans morsitans, Haplophilus subterraneus, Kirengeshoma palmata, Lysimachia japonica, Macrolophus pygmaeus, Microtus cabrerae, Mytilus galloprovincialis, Pallisentis (Neosentis) celatus, Pulmonaria officinalis, Salminus franciscanus, Thais chocolata and Zootoca vivipara. These loci were cross-tested on the following species: Acanthina monodon, Alytes cisternasii, Alytes maurus, Alytes muletensis, Alytes obstetricans almogavarii, Alytes obstetricans boscai, Alytes obstetricans obstetricans, Alytes obstetricans pertinax, Cambarellus montezumae, Cambarellus zempoalensis, Chorus giganteus, Cobitis tetralineata, Glossina fuscipes fuscipes, Glossina pallidipes, Lysimachia japonica var. japonica, Lysimachia japonica var. minutissima, Orconectes virilis, Pacifastacus leniusculus, Procambarus clarkii, Salminus brasiliensis and Salminus hilarii.
\end{abstract}

This article documents the addition of 238 microsatellite marker loci to the Molecular Ecology Resources Database. Table 1 contains information on the focal species, the number of loci developed, any other species the loci were tested in and the accession numbers for the loci in both the Molecular Ecology Resources Database and
GenBank. The authors responsible for each set of loci are listed in the final column. A full description of the development protocol for the loci presented here can be found on the Molecular Ecology Resources Database (http:// tomato.biol.trinity.edu/).

Table 1 Information on the focal species, the number of loci developed, any other species the loci were tested in and the accession numbers for the loci in both the Molecular Ecology Resources Database and GenBank. The authors responsible for each set of loci are listed in the final column

\begin{tabular}{|c|c|c|c|c|c|}
\hline Species & $\begin{array}{l}\text { No. primers } \\
\text { developed }\end{array}$ & Other species tested & $\begin{array}{l}\text { MER } \\
\text { database no. }\end{array}$ & GenBank accession no. & Authors \\
\hline $\begin{array}{l}\text { Alytes } \\
\text { dickhilleni }\end{array}$ & 13 & $\begin{array}{l}\text { A. obstetricans pertinax, } \\
\text { A. obstetricans obstetricans, } \\
\text { A. obstetricans boscai, } \\
\text { A. obstetricans almogavarii, } \\
\text { A. muletensis, A. maurus, } \\
\text { A. misternasii }\end{array}$ & $45209-45221$ & HQ693828-HQ693840 & $\begin{array}{l}\text { Carvalho, B. M.; Lopes, S.; } \\
\text { Van de Vliet, M. S.; Dias, } \\
\text { G.; Benítez, M.; Beltrán, J. } \\
\text { F.; Tejedo, M.; Ferrand, N.; } \\
\text { Gonçalves, H. }\end{array}$ \\
\hline
\end{tabular}


588 PERMANENT GENETIC RESOURCES NOTE

Table 1 Continued

\begin{tabular}{|c|c|c|c|c|c|}
\hline Species & $\begin{array}{l}\text { No. primers } \\
\text { developed }\end{array}$ & Other species tested & $\begin{array}{l}\text { MER } \\
\text { database no. }\end{array}$ & GenBank accession no. & Authors \\
\hline Arapaima gigas & 10 & $\mathrm{n} / \mathrm{a}$ & $45253-45262$ & HM013750-HM013759 & $\begin{array}{l}\text { Santos, Carlos Henrique } \\
\text { dos Anjos; Climaco, Gisele } \\
\text { Torres; Sousa, Carolina } \\
\text { Fernandes da Silva; } \\
\text { Paula-Silva, Maria Nazaré; } \\
\text { Sousa, Adna Cristina } \\
\text { Barbosa; Farias, Izeni } \\
\text { Pires; Campos, Tatiana; } \\
\text { Almeida-Val, Vera Maria } \\
\text { Fonseca }\end{array}$ \\
\hline $\begin{array}{l}\text { Austropotamobius } \\
\text { italicus }\end{array}$ & 12 & $\begin{array}{l}\text { Pacifastacus leniusculus, } \\
\text { Cambarellus zempoalensis, } \\
\text { C. montezumae, } \\
\text { Orconectes virilis, } \\
\text { Procambarus clarkii }\end{array}$ & $45393-45404$ & HQ593123-HQ593134 & $\begin{array}{l}\text { Pedraza-Lara, C.; } \\
\text { Gonzalez, E. G.; Bloor, P.; } \\
\text { Doadrio, I. }\end{array}$ \\
\hline $\begin{array}{l}\text { Blumeria graminis } \\
\text { f. sp. tritici }\end{array}$ & 9 & $\mathrm{n} / \mathrm{a}$ & $\begin{array}{l}45222,45223 \\
45225-45231 \\
\text { (see also } \\
45224)\end{array}$ & $\begin{array}{l}\text { HQ631364, } \\
\text { HQ631366-HQ631373 }\end{array}$ & $\begin{array}{l}\text { Parks, Ryan; Booth, } \\
\text { Warren; Cowger, Christina }\end{array}$ \\
\hline Cobitis lutheri & 11 & C. tetralineata & $45281-45291$ & HQ158597-HQ158607 & $\begin{array}{l}\text { Kwan, Ye-Seul; Lee, } \\
\text { Wan-Ok; Won, Yong-Jin }\end{array}$ \\
\hline $\begin{array}{l}\text { Dendroctonus } \\
\text { ponderosae }\end{array}$ & 50 & $\mathrm{n} / \mathrm{a}$ & $45343-45392$ & $\begin{array}{l}\text { GO486077, GT317345, } \\
\text { GT320845, GT322895, } \\
\text { GT324623, GT324841, } \\
\text { GT325939, GT328703, } \\
\text { GT331212, GT339861, } \\
\text { GT344705, GT345241, } \\
\text { GT350467, GT350767, } \\
\text { GT356832, GT357891, } \\
\text { GT363660, GT369500, } \\
\text { GT373329, GT381367, } \\
\text { GT383057, GT393905, } \\
\text { GT401041, GT403944, } \\
\text { GT404280, GT408450, } \\
\text { GT413070, GT413201, } \\
\text { GT415941, GT416554, } \\
\text { GT419741, GT421807, } \\
\text { GT429515, GT430043, } \\
\text { GT433817, GT436798, } \\
\text { GT451465, GT457678, } \\
\text { GT458184, GT461671, } \\
\text { GT464982, GT465588, } \\
\text { GT473994, GT474165, } \\
\text { GT485805, GT486724, } \\
\text { GT489170, GT490424, } \\
\text { GT490498, GT490735, } \\
\text { GT491361 }\end{array}$ & $\begin{array}{l}\text { Samarasekera, N Gayathri; } \\
\text { Keeling, Christopher I.; } \\
\text { Bohlmann, Jörg; Murray, } \\
\text { Brent W. }\end{array}$ \\
\hline $\begin{array}{l}\text { Glossina } \\
\text { morsitans } \\
\text { morsitans }\end{array}$ & 14 & $\begin{array}{l}\text { G. fuscipes fuscipes, } \\
\text { G. pallidipes }\end{array}$ & $45232-45252$ & See paper for details & $\begin{array}{l}\text { Hyseni, Chaz; Beadell, Jon } \\
\text { S.; Gomez Ocampo, Zaneli; } \\
\text { Ouma, Johnson O.; Okedi, } \\
\text { Loyce M.; Gaunt, Michael } \\
\text { W.; Caccone, Adalgisa }\end{array}$ \\
\hline $\begin{array}{l}\text { Haplophilus } \\
\text { subterraneus }\end{array}$ & 11 & $\mathrm{n} / \mathrm{a}$ & $45198-45208$ & HQ670723-HQ670733 & $\begin{array}{l}\text { Congiu, L.; Boscari, E.; } \\
\text { Bino, G.; Barbisan, F.; } \\
\text { Leśniewska, M.; Fusco, G. }\end{array}$ \\
\hline
\end{tabular}


Table 1 Continued

\begin{tabular}{|c|c|c|c|c|c|}
\hline Species & $\begin{array}{l}\text { No. primers } \\
\text { developed }\end{array}$ & Other species tested & $\begin{array}{l}\text { MER } \\
\text { database no. }\end{array}$ & GenBank accession no. & Authors \\
\hline $\begin{array}{l}\text { Kirengeshoma } \\
\text { palmata }\end{array}$ & 8 & $\mathrm{n} / \mathrm{a}$ & $45176-45183$ & $\begin{array}{l}\text { AB571675-AB571678, } \\
\text { AB571681, AB571682, } \\
\text { AB598398, AB598399 }\end{array}$ & $\begin{array}{l}\text { Yamashiro, T.; } \\
\text { Yamashiro, A. }\end{array}$ \\
\hline $\begin{array}{l}\text { Lysimachia } \\
\text { japonica }\end{array}$ & 10 & $\begin{array}{l}\text { L. japonica var. japonica, } \\
\text { L. japonica var. minutissima }\end{array}$ & $\begin{array}{l}45162,45163 \\
45331-45342\end{array}$ & AB591815-AB591824 & $\begin{array}{l}\text { Shinohara, Wataru; } \\
\text { Kakezawa, Akihiro; } \\
\text { Kawaguchi, Eri; Agata, } \\
\text { Kiyokazu }\end{array}$ \\
\hline $\begin{array}{l}\text { Macrolophus } \\
\text { pygmaeus }\end{array}$ & 10 & $\mathrm{n} / \mathrm{a}$ & $45264-45273$ & $\begin{array}{l}\text { HM208591-HM208599, } \\
\text { HQ853699 }\end{array}$ & $\begin{array}{l}\text { Sanchez, Juan A.; La Spina, } \\
\text { Michelangelo; Perera, } \\
\text { Omaththage P. }\end{array}$ \\
\hline Microtus cabrerae & 12 & $\mathrm{n} / \mathrm{a}$ & $45292-45303$ & $\begin{array}{l}\text { AF268902, AF268903, } \\
\text { EF666983, EF666984, } \\
\text { EF666987, EF666990, } \\
\text { EF666991, EU101013, } \\
\text { EU101014, EU101016, } \\
\text { EU101021, FR820649 }\end{array}$ & $\begin{array}{l}\text { Alasaad, Samer; Soriguer, } \\
\text { Ramón C.; Wandeler, } \\
\text { Peter; Jowers, Michael J.; } \\
\text { Sánchez, Antonio }\end{array}$ \\
\hline $\begin{array}{l}\text { Mytilus } \\
\text { galloprovincialis }\end{array}$ & 15 & $\mathrm{n} / \mathrm{a}$ & $45132-45146$ & $\begin{array}{l}\text { AJ625605, AJ626093, } \\
\text { AJ624322, AJ938131, } \\
\text { AJ938131, EH663192, } \\
\text { EH663098, EH663076, } \\
\text { EH662757, FL498494.1, } \\
\text { FL500528.1, FL498564.1, } \\
\text { FL495095.1, FL501296.1, } \\
\text { BV725482 }\end{array}$ & $\begin{array}{l}\text { Pardo, B.G.; Vera, M.; } \\
\text { Pino-Querido, A.; } \\
\text { Álvarez-Dios, J.A.; } \\
\text { Martínez, P. }\end{array}$ \\
\hline $\begin{array}{l}\text { Pallisentis } \\
\text { (Neosentis) } \\
\text { celatus }\end{array}$ & 11 & $\mathrm{n} / \mathrm{a}$ & $45324-45336$ & HQ588802-HQ588812 & $\begin{array}{l}\text { Li, Yang: Liu, Xiaolin: Liu, } \\
\text { Haixia; Wang, Weimin; } \\
\text { Zhu, Changcheng }\end{array}$ \\
\hline $\begin{array}{l}\text { Pulmonaria } \\
\text { officinalis }\end{array}$ & 8 & $\mathrm{n} / \mathrm{a}$ & $45304-45311$ & HQ452963-HQ452970 & $\begin{array}{l}\text { Meeus, S.; Honnay, O.; } \\
\text { Vandepitte, K.; } \\
\text { Jacquemyn, H. }\end{array}$ \\
\hline $\begin{array}{l}\text { Salminus } \\
\text { franciscanus }\end{array}$ & 10 & S. brasiliensis, S. hilarii & $\begin{array}{l}45184-45197 \\
\text { (includes } \\
\text { monomorphic } \\
\text { loci) }\end{array}$ & $\begin{array}{l}\text { HQ317313-HQ137316, } \\
\text { HQ137320-HQ137326 }\end{array}$ & $\begin{array}{l}\text { Rossini, Bruno C.; Nunes, } \\
\text { Aline G.; Freitas, Patrícia } \\
\text { D.; Galetti Jr, Pedro M. }\end{array}$ \\
\hline Thais chocolata & 12 & $\begin{array}{l}\text { Acanthina monodon, } \\
\text { Chorus giganteus }\end{array}$ & $45312-45323$ & HQ700360-HQ700371 & $\begin{array}{l}\text { Gallardo-Escárate, } \\
\text { CristianValenzuela- } \\
\text { Bustamante, Myriam; } \\
\text { Haye Pilar }\end{array}$ \\
\hline Zootoca vivipara & 12 & $\mathrm{n} / \mathrm{a}$ & $45164-45175$ & HQ337631-HQ337642 & $\begin{array}{l}\text { Stevens, Virginie M.; } \\
\text { Richard, Murielle; Bleay, } \\
\text { Colin; Clobert, Jean }\end{array}$ \\
\hline
\end{tabular}

